\title{
The projectors of the decomposition theorem are motivated
}

\author{
Mark Andrea de Cataldo And Luca Migliorini
}

\begin{abstract}
We prove that the projectors arising from the decomposition theorem applied to a projective map of quasi-projective varieties are absolute Hodge, André motivated, Tate and Ogus classes. As a by-product, we introduce, in characteristic zero, the notions of algebraic de Rham intersection cohomology groups of a quasiprojective variety and of intersection cohomology motive of a projective variety.
\end{abstract}

\section{Introduction and preliminary material}

P. Deligne introduced the notion of absolute Hodge classes on a smooth proper variety over a field $K$ of characteristic zero in [15], where he proved that Hodge classes on complex Abelian varieties are absolute Hodge. This is a powerful statement: on a complex Abelian variety, the notion of a Hodge class is purely algebraic. On a nonsingular proper complex variety as above, algebraic cycle classes are absolute Hodge, and absolute Hodge classes are Hodge classes. A positive answer to the Hodge conjecture would imply that these implications can be reversed. By considering absolute Hodge correspondences, one defines the category of absolute Hodge motives; see $\S 6$ of [17]. There is also a notion of absolute Hodge map. One can work with possibly singular varieties, where the notions of absolute Hodge cycles and maps are meaningful.

For a nonsingular projective $K$-variety, there is the stronger notion of "motivated cycle", due to Y. André [1]. The corresponding category of motives turns out to be Abelian, semi-simple and Tannakian. In terms of cycles, we have the implications: algebraic $\Longrightarrow$ motivated $\Longrightarrow$ absolute Hodge. A positive solution to the standard conjectures would reverse the first implication. A positive solution to the Hodge conjecture would reverse both. 
Let $K$ be an algebraically closed field of characteristic zero, let $f: X \rightarrow$ $Y$ be a projective map of quasi-projective $K$-varieties and let $E$ be an $f$ ample line bundle on $X$. In this paper, first we show how the decomposition and relative hard Lefschetz theorems [3, 12] give rise to self-maps of the intersection cohomology groups of $X$, the projectors of the decomposition theorem, and then we prove Theorem 2.5.1: these projectors are absolute Hodge maps. If $X$ is nonsingular projective, a stronger result, i.e. Theorem 2.6.2, holds: the projectors originating from the decomposition theorem are motivated cycles in $X \times X$, and the decomposition theorem holds at the level of André, hence absolute Hodge, motives. In particular, it follows that it is possible to define, for a singular projective variety, an André motive whose realization is intersection cohomology.

$\S 3$ is devoted to prove some variants of Theorem 2.5.1: the projectors are absolute Hodge over any field of characteristic zero, they are Tate classes and, finally, they are absolutely Hodge and Tate in the sense of A. Ogus.

There is no loss of generality in assuming that tr.deg. $K<\infty$; in particular, the field $K$ can be embedded into the field $\mathbb{C}$ of complex numbers. The notion of absolute Hodge involves the interplay of three cohomology theories: de Rham, étale $\mathbb{Q}_{\ell}$-adic and, after base change via an embedding of the field into $\mathbb{C}$, Betti.

We take as starting point the decomposition and relative Hard Lefschetz theorems in the Betti theory and, from that point on, we work exclusively in cohomology, i.e. we make no further use of derived categories. There are three reasons for this. The first is that we are not aware of the existence in the literature of the cup-product operation at the level of the derived category of D-modules (de Rham side). The second is that in the contexts of motivated cycles and of crystalline cohomology, such derived techniques do not seem to be available at the present time. The third is that we found working within the context of cohomology and of its fundamental functoriality properties aesthetically pleasing.

The key point in all the results of this paper, is to construct the projectors of the decomposition theorem in a uniform way in all cohomology theories, so that they turn out to be, more or less automatically, compatible with each other via the comparison isomorphisms. We employ four main $K$-rational constructions from $\S 1.3$ : a geometric construction of the perverse filtration; stratifications of maps; linear algebra description of the decomposition by supports; splittings in abelian categories. First, we work with $X$ nonsingular, then we refine our analysis to the singular case. Along the way, we offer in $\S 2.3$ a definition of intersection de Rham cohomology as a certain 
subquotient of the de Rham cohomology of a resolution, and we point out several of its properties.

We thank F. Charles and to B. Bhatt for useful conversations. We thank the anonymous referee for the thoughtful suggestions.

\subsection{Notation, conventions and some preliminary facts}

The set-up. Unless otherwise stated, we work over an algebraically closed field $K$ of finite transcendence degree over $\mathbb{Q}$. A $K$-scheme is a separated scheme of finite type over $K$; a $K$-variety is an integral $K$-scheme. The restriction on the transcendence degree of $K$ is for convenience of exposition only; see $\S 3.1$. The embeddings of $K$ into $\mathbb{C}$ are denoted by $\sigma$. If $X$ is a $K$ scheme, we denote by $\sigma X$ the pull-back to $\mathbb{C}$ via $\sigma$; similarly, for $K$-maps, etc.

We employ the following cohomology theories (see [15]): $H_{d R}(-/ K)$ (de Rham), $H_{e t}\left(-, \mathbb{Q}_{\ell}\right)\left(\mathbb{Q}_{\ell}\right.$-adic); if $K=\mathbb{C}$, then we have $H_{B}(-,-)$ (the

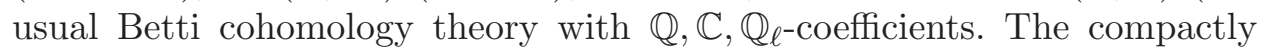
supported counterparts are denoted $H_{!, d R}(-/ K)$, etc. We fix the following data:

$$
h:=f \circ g: W \stackrel{g}{\longrightarrow} X \stackrel{f}{\longrightarrow} Y, \quad \eta:=c_{1}(E),
$$

where $f, g$ are projective maps of quasi-projective $K$-schemes, $E$ is an $f$ ample line bundle on $X$ and $\eta:=c_{1}(E) \in H^{2}(X)(1)$, in any of the cohomology theories that we shall employ. Our interest actually lies in the datum of $(f, \eta)$. The map $g$ is usually going to be a resolution of the singularities of $X$, and it is introduced as means to reduce proofs of statements about $(f, \eta)$ to the case when $X$ is nonsingular.

Conventions on cohomological and filtration degrees. Since the bookkeeping of cohomological and filtration degrees is inessential and a distraction, we omit it for the most part. Here is the list of the conventions that, unless mentioned otherwise, we employ implicitly: the cohomology groups $H^{k}(X)$ live in the range $[0,2 \operatorname{dim} X]$, the Hodge filtrations $\mathscr{F}$ (Betti and de Rham) in the range $[0, \operatorname{dim} X]$, and the weight filtrations $\mathscr{W}$ (all three theories) in the interval $[0,2 \operatorname{dim} X]$; if $X$ is integral and nonsingular, then the Betti intersection complex is $I C_{X}=\mathbb{Q}_{X}[\operatorname{dim} X]$; if $X$ is irreducible, then the cohomology sheaves $\mathcal{H}^{k}\left(I C_{X}\right)$ live in the range [- $\left.\operatorname{dim} X,-1\right]$ and the intersection cohomology groups $I H^{k}(X):=\mathbb{H}^{k-\operatorname{dim} X}\left(I C_{X}\right)$ live in the same range as cohomology, i.e. [0,2 $\operatorname{dim} X]$; for general $X$, we write $X=\cup X_{i}$ (union of irreducible components), form the finite map $\nu: \coprod_{i} X_{i} \rightarrow X$ and set $I C_{X}:=$ 
$\nu_{*}\left(\oplus_{i} I C_{X_{i}}\right)$; we place the groups $I H^{k}\left(X_{i}\right)$ in the range $\left[0,2 \operatorname{dim} X_{i}\right]$ ([7], $\S 4.6)$; the perverse filtration $\mathscr{P}_{f}$ on $I H(X)$ lives in the range $[-r(f), r(f)]$, where $r(f)$ is a convenient integer, called the defect of semismallness of $f$ in $[10,12]$. We employ the same conventions for $H_{!}(X)$ and $I H_{!}(X)$.

Decomposition, relative hard Lefschetz and semisimplicity theorems. We refer to the survey [12] for the language and facts surrounding these theorems, which we use freely in what follows, especially in $\S 1.4$.

Supports. A semisimple perverse sheaf $P$ (étale or Betti) on a $K$-scheme $V$ splits canonically $P \cong \bigoplus_{\mathfrak{V} \in \mathcal{S}(P)} P_{\mathfrak{V}}$ as the finite direct sum, over a finite set $\mathcal{S}(P)$ of distinct integral subvarieties $\mathfrak{V} \subseteq V$, of the intersection complexes $P_{\mathfrak{V}}$ of the varieties $\mathfrak{V}$ with suitable twisted semisimple coefficients. We call $\mathcal{S}(P)$ the set of supports of $P$. Let $\phi: U \rightarrow V$ be a projective map of quasi-projective $K$-schemes, and let $Q$ be a semisimple complex of geometric origin ([3], 6.2.4), e.g. $Q=I C_{U}$. By the decomposition theorem, we have a non-canonical finite direct sum decomposition: $\phi_{*} Q \cong \bigoplus_{a} Q_{\phi, a}[-a]$, where $Q_{\phi, a}:={ }^{\mathfrak{p}} R^{a} \phi_{*} Q$ (perverse direct image sheaf). Each $Q_{\phi, a}$ is a semisimple perverse sheaf and we obtain $\mathcal{S}(\phi, Q, a):=\mathcal{S}\left(Q_{\phi, a}\right)$, the set of supports of $\phi_{*} Q$ in perversity $a$. The cohomology groups $H(U, Q)=H\left(V, \phi_{*} Q\right)$ are filtered by the perverse filtration $\mathscr{P}_{\phi}$ and, given that $Q$ will be fixed by the context, we set $G r_{\phi, a}:=G r_{a}^{\mathscr{P}_{\phi}} H(U, Q)$ and we denote the resulting decomposition by supports by $G r_{\phi, a}=\oplus_{\mathfrak{V} \in \mathcal{S}(\phi, Q, a)} G r_{\phi, a, \mathfrak{V}}$.

Comparison Betti-de Rham and Betti-étale. The algebraic de Rham cohomology groups $H_{d R}(X / K)$ for quasi-projective nonsingular varieties were defined in [19] and they where proved to be isomorphic to the complex Betti cohomology groups when $K=\mathbb{C}$. The extension to singular varieties and the definition of Borel-Moore de Rham homology were introduced and studied by Hartshorne in [20]. Applying the simplicial methods of [14] and the comparison theorem with Betti cohomology endows $H_{d R}(X / K)$ with the two natural filtrations $\mathscr{F}$ (Hodge) and $\mathscr{W}$ (weight). The usual maps between algebraic de Rham cohomology groups, e.g. pull-backs via algebraic maps, are filtered strict for these filtrations. Recall that a filtered map is strict if taking graded objects is exact. For any embedding $\sigma$ of $K$ into $\mathbb{C}$, there are the natural comparison isomorphisms $H_{B}(\sigma X, \mathbb{C}) \cong H_{d R}(\sigma X / \mathbb{C})$ which are bi-filtered strict for $\mathscr{F}$ and $\mathscr{W}$. Similarly, we have the natural comparison isomorphisms $H_{B}\left(\sigma X, \mathbb{Q}_{\ell}\right) \cong H_{e t}\left(\sigma X, \mathbb{Q}_{\ell}\right)$ which is filtered strict for the weight filtrations on both sides. All of the above applies to $H_{\text {! }}$. 


\subsection{Motivated endomorphism in cohomology}

In this section we define the notion of motivated endomorphism, which plays a crucial role in this paper. Our main results assert that the projectors arising from the decomposition theorem are motivated. When $X$ is nonsingular projective the strong notion of André motivated cycle, recalled in Section 1.2.1 is available, while, if $X$ is only quasi-projective, we need to consider the a-priori weaker notion of absolute Hodge cycle, whose definition we recall in Section 1.2.2.

1.2.1. André motivated endomorphisms. We recall the definition of Y. André's motivated cycles $A_{\text {mot }}^{\bullet}(X)$ and the corresponding category of motives $\mathcal{M}_{K}$; see [1], $\S 4$. Let $K$ be an algebraically closed field of characteristic zero and let $X$ be a nonsingular projective $K$-variety of dimension $n$. Given an ample line bundle defined over $K$, with first Chern class $\eta \in H_{d R}^{2}(X / K)$, we denote by $L$ the Lefschetz operator on de Rham cohomology defined by cupping with $\eta$. We recall the definition of the Lefschetz involution operator $*_{L}$ : if $\alpha$ is a degree $j$ cohomology class, then $*_{L} \alpha=L^{n-j} \alpha$ see [1], p.10. Roughly speaking, a motivated cycle is a cohomology class on the projective nonsingular variety $X$ which can be obtained applying standard operations on algebraic cycle classes and the Lefschetz involution operator. More precisely, a degree $d$ cohomology class $\xi \in H_{d R}^{2 d}(X / K)(d)$ is motivated if there exists a nonsingular projective $K$-variety $Y$, algebraic cycles $A, B$ in $X \times{ }_{K} Y$, with cohomology classes $\alpha, \beta \in H_{d R}(X \times Y / K)$ such that

$$
\xi=p_{1 *}(\alpha \cup * \beta)
$$

where $p_{1}, p_{2}$ are the projection on the two factors and $*$ denotes the Lefschetz involution with respect to a "product" polarization $p_{1}^{*} \eta_{1} \otimes p_{2}^{*} \eta_{2}$ on $X \times Y$. We denote by $A_{\text {mot }}^{\bullet}(X)$ the vector sub-space of motivated classes on $X$ and by $c l_{H}: A_{\text {mot }}^{\bullet}(X) \rightarrow H_{d R}^{2 \bullet}(X / K)(\bullet)$ the natural injection. The category of André motives $\mathcal{M}_{K}$-defined the same way as Grothendieck's, but using motivated correspondences in lieu of algebraic ones- is Tannakian, graded, abelian semi-simple and polarizable ([1], Thm. 0.4). Let $h(X) \in \mathcal{M}_{K}$ denote the object (André motive) associated with a smooth projective $K$-variety $X$. Since the Künneth components of the diagonal are motivated classes, we have $h(X)=\oplus_{d} h^{d}(X)$. The de Rham realization functors realizes $h^{d}(X)$ as $H_{d R}^{d}(X / K)$, together with its Hodge filtrations. We have $\operatorname{End}_{\mathcal{M}_{K}}(h(X))=$ $A_{\mathrm{mot}}^{\operatorname{dim}} X(X \times X) \subseteq_{c l_{H}} H_{d R}^{2 \operatorname{dim} X}(X \times X / K)(\operatorname{dim} X)$. Note that the last term lives in $\operatorname{End}^{o}\left(H_{d R}(\bar{X} / K)\right)$. In $\S 2.6$, where we prove that the projectors associated with the decomposition theorem for a projective map are motivated, 
we do not use the Definition (2) of motivated cycles directly, but rather only the formal properties of the category $\mathcal{M}_{K}$ stated above.

Definition 1.2.1. An endomorphism in $\operatorname{End}^{\circ}\left(H_{d R}(X / K)\right)$ is André motivated if it lies in the image of $A_{\operatorname{mot}}^{\operatorname{dim} X}(X \times X)$.

Via $c l_{H}$, a motivated codimension $p$ cycle gives rise to a cycle in $H_{d R}^{2 p}(X / K)(p)$. If $K$ is embeddable in $\mathbb{C}$, then such a cycle gives rise to an absolute Hodge class ([1], Proposition 2.5.1). Conversely, absolute Hodge cycles are expected to give rise to motivated classes.

1.2.2. Absolute Hodge endomorphism. Absolute Hodge classes are collections of cohomology classes in the different cohomology theories available (Betti, de Rham and étale $\mathbb{Q}_{\ell}$-adic in our case), which enjoy strong compatibility properties with respect to comparison isomorphisms, change of coefficients and the base change maps corresponding to the different $\mathbb{C}$ imbeddings of $K$. We may summarize these compatibilities in a diagram:

Let $(K, X, \sigma)$ be as in (1). We have

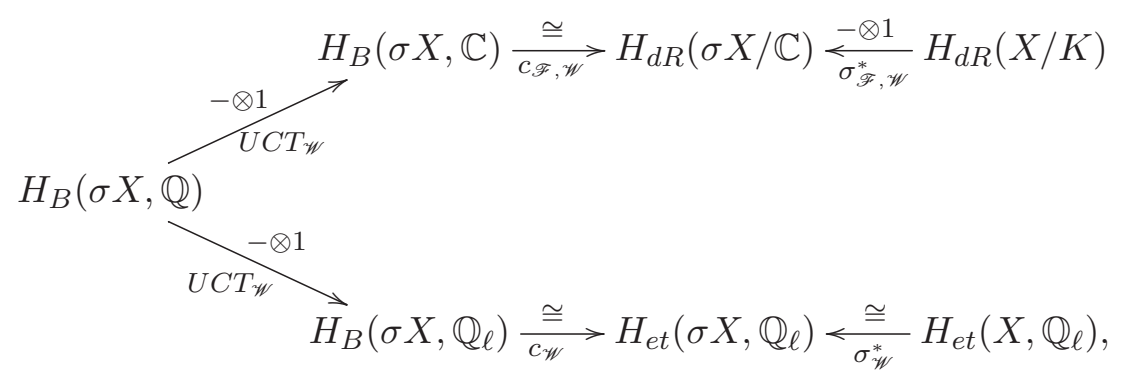

where the arrows UCT come from the universal coefficient theorem; the arrows $c$ are the comparison isomorphism for Betti-de Rham and Betti-étale cohomology; the arrows $\sigma^{*}$ come from base change via $\sigma$; the arrows are strictly compatible for the indicated filtrations.

Define:

$$
\underline{H}(X):=H_{d R}(X / K) \times \prod_{\ell} H_{e t}\left(X, \mathbb{Q}_{\ell}\right) .
$$

We define $\underline{H}(\sigma X)$ in a similar way. There are natural maps $\left(\delta_{\mathbb{Q}}\right.$ : product of comparison maps $(3) ; \sigma^{*}$ : base change via $\left.\sigma\right)$ :

$$
H_{B}(\sigma X, \mathbb{Q}) \stackrel{\delta_{\mathbb{Q}}}{\longrightarrow} \underline{H}(\sigma X) \stackrel{\sigma^{*}}{\stackrel{H}{H}(X) .}
$$


We can replace $H(X)$ with $H(X)(m)$ or with, say, the degree-preserving endomorphisms $\operatorname{End}^{o}(H(X))$ of $H(X)$. We thus obtain diagram $(*)$, as well as the analogue of $(5)$, for $\operatorname{End}^{o}(H(X))$, and these are the two ingredients needed for the following:

Definition 1.2.2. We say that $\underline{\zeta}=\left(\zeta_{d R},\left\{\zeta_{\text {et }}\right\}\right) \in \underline{H}^{2 p}(X)(p)$ is absolute Hodge if:

1) $\sigma^{*} \zeta \in \operatorname{Im} \delta_{\mathbb{Q}}$ (rationality), for every $\sigma$;

2) $\zeta_{d R} \in\left(\mathscr{F}^{0} \cap \mathscr{W}_{0}\right) H_{d R}^{2 p}(X / K)(p)$.

Similarly, for $\underline{\zeta} \in \underline{\operatorname{End}}^{o}(H(X))$.

Remark 1.2.3. Membership of $\zeta_{d R} \in\left(\mathscr{F}^{0} \cap \mathscr{W}_{0}\right) \operatorname{End}^{o}\left(H_{d R}(X / K)\right)$ means that $\zeta_{d R}$ preserves the Hodge and weight filtrations on $H_{d R}(X / K)$. In the Betti context, membership in $\left(\mathscr{F}^{0} \cap \mathscr{W}_{0}\right) \operatorname{End}^{o}\left(H_{B}(X, \mathbb{Q})\right)$ is equivalent to the map being a morphism of rational mixed Hodge structures.

A $K$-map $f: X \rightarrow Y$ of $K$-varieties defines pull-backs maps from all the vertices of the diagram (3) for $Y$ to those of the diagram for $X$, where all the squares are commutative. From such map of diagrams one can form kernels, images and cokernels, which still fit in the comparison diagram 3. An essential step in several proofs in this paper consists in showing that some linear algebra construction, such as taking a subquotient defined by the perverse filtration associated to a map, or projecting on a graded summand of the direct sum decompositions (13) and (14), can be expressed in such a way that it fits in a diagram like (3). We introduce a special terminology for this:

Definition 1.2.4. (Compatibility diagram $(*)$ ) Let $V$ be a direct sum of tensor products of Tate-twisted subquotients of $H(X)$, obtained via some construction carried out on the various vertices of the diagram 3 . We say that we have the compatibility diagram $(*)$ for $V$ if, for every $\sigma$, we can replace $H(X)$ with $V$ at all the vertices in (3) and retain all the properties listed above of the resulting arrows induced by the construction. Similarly, given a map $u: V \rightarrow V^{\prime}$ between two such objects, obtained via some construction carried out at each vertex, we say that we have the compatibility diagram (*) for $u$ if we have compatibility diagrams $(*)$ for $V$ and $V^{\prime}$ and the arrows $u$ yield a map of the compatibility diagrams $(*)$ that makes all squares commutative and such all the arrows between corresponding vertices of the two compatibility diagrams $(*)$ are filtered strict for the indicated filtrations. 
The gist of the above definition is that a given construction $u: V \rightarrow V^{\prime}$, when seen at the de Rham vertex corresponding to $\sigma X$, is $\mathbb{Q}$-rational as well as $K$-rational.

In Proposition 2.1.1 we show that we have compatibility diagrams (*) for the subspaces of the perverse filtration $\mathscr{P}_{f}$ and hence for its graded pieces $G r_{f}:=G r^{\mathscr{P}_{f}}$. Similarly, Propositions 2.2.1, 2.4.1 show that we have compatibility diagrams $(*)$ for the direct sum decompositions (13) and for the splitting (14).

\subsection{Three geometric constructions and a linear algebra splitting}

1.3.1. The filtrations induced by a flag. Let $(K, f)$ be as in (1). Our aim is to define the "flag" filtrations (7) on the cohomology of the domain of a map of varieties. The definition is geometric in the sense that it is based on the diagram of maps (6). For a general flag, these flag filtrations coincide with the perverse Leray filtration, so that the latter admits a geometric description. It is this latter that plays a central role in this paper.

Form a Cartesian diagram of $K$-varieties:

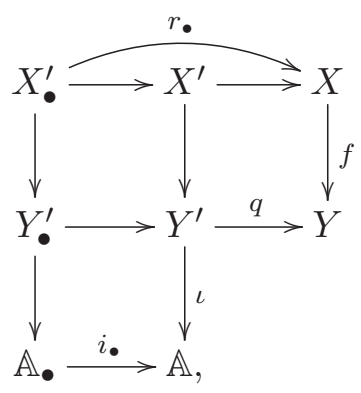

where

1) $q$ is any map subject to the following properties: $Y^{\prime}$ is affine, $q$ is a Zariski locally trivial $\mathbb{A}^{d}$-fibration for some $d \geq 0$; the existence of $q$, which is never unique, is ensured by the quasi-projectivity of $Y$ ("Jouanolou's trick" [7]);

2) $\iota$ is any closed embedding into an affine space $\mathbb{A}$ of some dimension $N$;

3) $i_{\text {• }}$ is the embedding of a complete flag of affine linear sections of $\mathbb{A}$ :

$$
\mathbb{A}_{\bullet}=\left\{\emptyset=: \mathbb{A}_{-(N+1)} \subseteq \cdots \subseteq \mathbb{A}_{0}:=\mathbb{A}\right\},
$$

where $\mathbb{A}_{-c}$ is a codimension $c$ linear subspace of $\mathbb{A}$. 
The left-most-side of this diagram corresponds to a choice of a point $\beta \in \mathcal{B}(K)$, where $\mathcal{B}$ is the variety of complete affine linear flags in $\mathbb{A}$. For any such $\beta \in \mathcal{B}(K)$, define increasing filtrations $F^{\beta}$ on $H(X)$ and on $H_{!}(X)$ by setting: $\left(r_{!, k}\right.$ shifts cohomological degrees)

$$
\begin{gathered}
F_{k}^{\beta} H(X):=\operatorname{Ker}\left\{H(X) \stackrel{r_{-k}^{*}}{\longrightarrow} H\left(X_{-k}^{\prime}\right)\right\}, \\
F_{!, k}^{\beta} H_{!}(X):=\operatorname{Im}\left\{H_{!}\left(X_{k}^{\prime}\right) \stackrel{r_{!, k}}{\longrightarrow} H_{!}(X)\right\} .
\end{gathered}
$$

Fact 1.3.1. (Perverse=general flag) According to [7], Theorem 3.3.5, for general $\beta \in \sigma \mathcal{B}(\mathbb{C})$, the filtrations of type $F_{B}^{\beta}, F_{!, B}^{\beta}$ appearing at every Betti vertex of the compatibility diagram (*) 3) for $H(X)$ and $H_{!}(X)$ coincide, up to a re-numbering of the cohomological degree and of the filtration index, with the perverse filtrations $\mathscr{P}_{B, f}$.

1.3.2. Teissier and Whitney stratifications of a map. The goal of this section is to prove Proposition 1.3.2 by using beautiful results of Verdier [25] and Teissier [24]. For background on stratifications, we refer to [18], p.43.

Let $(K, f)$ be as in (1). If $K=\mathbb{C}$, then there are the notions of: Whitney stratification of the complex analytic space underlying a $\mathbb{C}$-scheme; Whitney stratification of a map of $\mathbb{C}$-schemes, which requires the topological local triviality of $f$ over each stratum. Verdier has proved in [25], 3.3, 4.14-15, that one can produce algebraic Whitney stratifications, $\mathscr{T}_{X}: X=\coprod_{i} T_{i}^{\prime}$ and $\mathscr{T}_{Y}: Y=\coprod_{j} T_{j}$, of the map $f$ so that the strata $T$ are $\mathbb{C}$-subvarieties (locally closed, integral and nonsingular). This is achieved as follows: Verdier first proves that there are algebraic Whitney stratifications of $Y$ and $X$ (this is the hard part); it is then easy to refine algebraically both stratifications so that the following condition is met: every stratum on $X$ maps smoothly and surjectively onto a stratum on $Y$; at this point, $f$ is a stratified submersion for the refined algebraic Whitney stratifications; the Thom isotopy lemmata [18] imply the desired local triviality assertion.

Teissier has introduced a local algebraic condition on a stratification of a $\mathbb{C}$-scheme that, strikingly, implies that the stratification is a Whitney one: for the condition and the proof of the implication, see [24], Proposition III.2.3.1, p.398. Teissier's algebraic condition can be defined over any field, where it can be achieved by a simple noetherian induction based on the argument of the proof of [24], VI.2.1, p.477.

Given a stratification $\mathscr{T}_{Y}: Y=\coprod T_{i}$, denote by $\mathcal{S}_{\mathscr{T}_{Y}}$ the set of the closures $\overline{T_{i}} \subseteq Y$. 
Proposition 1.3.2. Let $(K, f)$ be as in 1). There is a stratification $\mathscr{T}_{Y}$ : $Y=\coprod T_{j}$ such that for every embedding $\sigma$ of $K$ into $\mathbb{C}$ and for every $b \in \mathbb{Z}$, the sets of supports $(\S 1.1) \mathcal{S}_{B}\left(\sigma f, b, I C_{B, \sigma X}\right) \subseteq \sigma\left(\mathcal{S}_{\mathscr{T}_{Y}}\right)$. In particular the supports of every $\sigma f$ are $K$-rational.

Sketch of proof. Start with a stratification of $Y$ subject to Teissier's conditions. Do the same for $X$. Refine both stratifications so that the following condition is met: every stratum on $X$ maps smoothly and surjectively onto a stratum on $Y$. Call $\mathscr{T}_{Y}$ and $\mathscr{T}_{X}$ the results, which automatically satisfy Teissier's conditions. By passing to $\mathbb{C}$, the discussion above shows that the $\sigma \mathscr{T}$ form a Whitney stratification of $\sigma f$. The local triviality assertion implies that the direct image complex $\sigma f_{*} I C_{B, \sigma X}$ is constructible with respect to $\sigma \mathscr{T}_{Y}$, so that the supports of $\sigma f_{*} I C_{\sigma X}$ are to be found in $\sigma \mathcal{S}_{\mathscr{T}_{Y}}$.

Remark 1.3.3. In a similar context, the rationality of the notion of Whitney stratification, as well as its relevance to motivic questions is pointed out in [2], Lemma 3.1.9, where Teissier's result is used to prove that the higher direct images by a $K$-map of a $K$-constructible sheaf are still $K$ constructible.

1.3.3. Contributions of strata: results from $[7,10]$. In this section, we introduce the $K$-rational construction (8); this is to be used later in the proof of Proposition 2.2.1. We specialize to $K=\mathbb{C}$ in order to recall, in the Betti context, the linear algebra characterization (12) of the summands corresponding to different supports in the decomposition (13). This characterization follows from the Claim on p.745 in [10], and Theorem 4.3.2 and Lemma 4.5.3 in [7].

Let $(K, f)$ be as in (1). Since we are going to use the construction that follows for $f$, as well for auxiliary maps $\phi: U \rightarrow V$, we use $\phi$ in what follows. Fix a stratification $\mathscr{T}_{V}$ of $V$ as in Proposition 1.3.2. For every stratum $T \subseteq V$, form the commutative diagram:

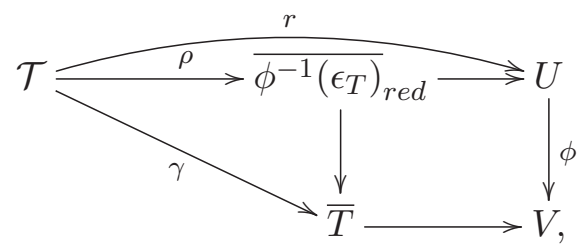

where $\bar{T} \rightarrow V$ is the closed embedding; $\epsilon_{T}$ is the generic point of $T$; ${\overline{\phi^{-1}\left(\epsilon_{T}\right)_{\text {red }}}}_{\rightarrow} \rightarrow$ is the closed embedding; $\rho$ is a proper, generically finite 
surjection from a nonsingular quasi-projective $K$-scheme, e.g. first normalize, then resolve the irreducible components; the proper map $r$ is the evident compositum. Since $r$ is proper, we have the pull-back maps:

$$
r^{*}: H(U) \longrightarrow H(\mathcal{T}), \quad r_{!}^{*}: H_{!}(U) \longrightarrow H_{!}(\mathcal{T})
$$

Notation. In the remainder of this section: $K=\mathbb{C}$; we keep the stratification $\mathscr{T}_{V}$ and we assume, in addition that $U, V$ are quasi-projective varieties with $U$ nonsingular of some dimension $d$; by cohomology we mean Betti cohomology with rational coefficients and we drop the Betti decoration; for $j \in \mathbb{Z}$, define the complementary degree $j^{\prime}:=2 d-j$ and denote by $\int_{U}: H^{j}(U) \times H_{!}^{j^{\prime}}(U) \rightarrow \mathbb{Q}$ the Poincaré duality pairing; finally, set $G r_{\phi, b}^{j}:=$ $G r_{\phi, b} H^{j}(U), G r_{!, \phi, b}^{j}:=G r_{\phi, b} H_{!}^{j}(U)$, and similarly for $G r_{\phi, b, \mathfrak{V}}^{j}, G r_{!, \phi, b, \mathfrak{V}}^{j}$.

The supports $\mathfrak{V}$ of $\phi$ are among the closures $\bar{T}$ of the strata. Note that what follows applies to all the strata $T$, not just to the ones appearing as supports in a given perversity. This is simply because, in that case, the corresponding terms are automatically zero (see [10]).

Recalling our conventions on the perverse filtration, the pull-back map in cohomology $r^{*}: H(U) \longrightarrow H(\mathcal{T})$ is compatible with the perverse filtrations, provided we shift the one for $\gamma$ by the fixed amount $d-\operatorname{dim} \mathcal{T}$. We set, accordingly, $b^{\prime}:=b+d-\operatorname{dim} \mathcal{T}$. Similarly, for the pull-back map $r_{!}^{*}$ for compact supports.

The map $r^{*}\left(r_{!}^{*}\right.$, resp. $)$ thus descend to a map $r_{b}^{*}: G r_{\phi, b} \rightarrow G r_{\gamma, b^{\prime}}\left(r_{!, b}^{*}\right.$, resp.) on the graded pieces, compatibly with the decomposition by supports, i.e. $r_{b}^{*}=\sum_{\mathfrak{V}} r_{b, \mathfrak{V}}^{*}\left(r_{!, b}^{*}=\sum_{\mathfrak{V}} r_{!, b, \mathfrak{V}}^{*}\right.$, resp. $)$. Note that the supports for the maps $\phi$ and $\gamma$ may be quite different, but this does not spoil the picture.

Denote by $r_{b, \bar{T}}^{*}: G r_{\phi, b} \rightarrow G r_{\gamma, b^{\prime}, \bar{T}}$ the compositum of the map $r_{b}^{*}$ followed by the projection onto the (possibly trivial) $\bar{T}$-th direct summand of $G r_{\gamma, b^{\prime}}$. Similarly, we have the map $r_{!, b, \bar{T}}^{*}: G r_{!, \phi, b} \rightarrow G r_{!, \gamma, b^{\prime}, \bar{T}}$.

The duality pairing descends to a non-degenerate pairing on the graded pieces:

$$
\int_{U}^{G r}: G r_{\phi, b}^{j} \times G r_{!, \phi,-b}^{j^{\prime}} \longrightarrow \mathbb{Q}, \quad(\alpha, \beta) \longrightarrow \int_{U}^{G r}(\alpha, \beta)
$$

and the $G r_{\phi, b, \mathfrak{V}}^{j}$ and $G r_{!, \phi,-b, \mathfrak{V}^{\prime}}^{j^{\prime}}$ are mutually orthogonal when $\mathfrak{V} \neq \mathfrak{V}^{\prime}$.

We can now state the desired characterization $[7,10]$ of the summand corresponding to the dense stratum $V^{o}$ : 


$$
\begin{aligned}
G r_{\phi, b, V} & =\bigcap_{S \neq V^{o}} \operatorname{Ker} r_{b, \bar{S}}^{*} \subseteq G r_{\phi, b}, \\
G r_{!, \phi, b, V} & =\bigcap_{S \neq V^{o}} \operatorname{Ker} r_{!, b, \bar{S}}^{*} \subseteq G r_{!, \phi, b},
\end{aligned}
$$

while, for the non-dense strata, the orthogonality property discussed above gives:

$$
G r_{\phi, b, \bar{T}}=\left(\bigcap_{S \neq T, V^{o}} \operatorname{Ker} r_{b, S}^{*}\right) \bigcap\left(G r_{!, \phi, b, V}\right)^{\perp \int_{U}^{G r}}
$$

The formula for $G r_{!, \phi, b, \bar{T}}$ is analogous.

1.3.4. HL-triples and splitting. Proposition 1.3.4 below is one of the keys to the cohomological approach in this paper: it singles out the preferred splitting in cohomology we work with; see (20) and Proposition 2.2.1. It should be compared with [16], Proposition 2.4, which is stated and proved at the level of triangulated categories with t-structures. By taking cohomology, the latter implies the former, in the Betti and étale contexts. On the other hand, Proposition 1.3.4 becomes useful in contexts where it is not immediately clear how to use triangulated categories (e.g. the cup product operation in the derived category of $D$-modules, in de Rham case), or where such formalism is absent (e.g., to our knowledge, crystalline cohomology).

Let $\mathcal{A}$ be an Abelian category endowed with an additive and exact autoequivalence $A \mapsto A(1)$, whose iterates are denoted by $(m)$, and let $\mathcal{A} F$ be the associated category of objects endowed with a finite increasing filtration. Given $(H, F) \in \mathcal{A} F$, we denote by $G r_{i} H:=G r_{i}^{F} H$, and by $\left(G r_{*} H, F\right)$ the associated filtered graded object, i.e. $G r_{*} H:=\oplus_{i} G r_{i} H$ with the "direct sum" filtration $F_{i} G r_{*} H:=\oplus_{a \leq i} G r_{a} H$. Let $(H, F, e)$ be a triple with $(H, F)$ in $\mathcal{A} F$ and $e: H \rightarrow H(1)$ subject to $e: F_{i} H \rightarrow F_{i+2} H(1)$. Assume that the triple $(H, F, e)$ is an $H L$-triple, i.e. that the induced maps $e^{i}: G r_{-i} H \rightarrow$ $G r_{i} H(i)$ are isomorphisms (hard Lefschetz property). We have the usual primitive Lefschetz decomposition $G r_{*} H=\oplus_{0 \leq j \leq i} P_{-i}^{e}(-j)$, where $P_{-i}^{e}:=$ Ker $e^{i+1} \subseteq G r_{-i} H$.

Proposition 1.3.4. ([8], Lemma 2.4.1) Let $(H, F, e)$ and $\left(G r_{*} H, F\right)$ be as above. There is a unique map $f_{i}: P_{-i}^{e} \rightarrow F_{-i} H$ with the following two properties: 
1) $f_{i}$ induces the natural inclusion $P_{-i}^{e} \rightarrow G r_{-i} H$;

2) $e^{s} \circ f_{i}: P_{-i}^{e} \rightarrow G r_{s} H(s)$ is zero $\forall s>i$.

The map $\phi_{e}:=\sum_{0 \leq j \leq i} e^{j} \circ f_{i}:\left(G r_{*} H, F\right) \rightarrow(H, F)$ is an isomorphism in $\mathcal{A} F$.

\subsection{The decomposition theorem and Betti cohomology}

Let $(f, g, \eta)$ be as in (1), except that we work over $\mathbb{C}$ and with rational Betti cohomology. Recall the notation in $\S 1.1$, especially the one for the graded pieces of the perverse filtration, and the one in $\S 1.3 .4$ for HL-triples. The analogous statements and constructions for compact supports are left implicit; e.g. write $G r_{! B, f}$ in $(13)$ and $\pi_{!, B}(f, \eta, b)$ in (16).

1.4.1. Decompositions associated with a projective map. If we set $Q:=I C_{X}$, then we have the finite direct sum decomposition by supports:

$$
I H_{B}(X) \cong \bigoplus_{b} \bigoplus_{\mathfrak{Y} \in \mathcal{S}(f, b, Q)} G r_{B, f, b, \mathfrak{Y}}
$$

The perverse Leray filtration $\mathscr{P}_{B, f}$ induced by $f$ on $I_{B}(X)$ is given by mixed Hodge substructures for the natural mixed Hodge structure on $\operatorname{IH}_{B}(X)$ constructed in [7], i.e. it is compatible with $\mathscr{W}$ and, after passing to $\mathbb{C}$ coefficients, with $\mathscr{F}$.

The relative Hard Lefschetz theorem implies that we have direct sum isomorphisms $\eta_{B}^{b}: \oplus_{\mathfrak{Y}} G r_{B, f,-b, \mathfrak{Y}} \cong \oplus_{\mathfrak{Y}} G r_{B, f, b, \mathfrak{Y}}(b)$ of mixed Hodge structures. In particular, we deduce that the triple $\left(I H_{B}(X), \mathscr{P}_{B, f}, \eta_{B}\right)$ is an HL-triple (§1.3.4) for the category of mixed Hodge structures. We obtain the following four decompositions of mixed Hodge structures:

$$
\begin{aligned}
G r_{B, f} & :=\bigoplus_{b} G r_{B, f, b}=\bigoplus_{b, \mathfrak{Y}} G r_{B, f, b, \mathfrak{Y}}=\bigoplus_{0 \leq j \leq i} P_{B,-i}^{\eta}(-j) \\
& =\bigoplus_{b} \bigoplus_{\mathfrak{Y},-i+2 j=b} P_{B,-i, \mathfrak{Y}}^{\eta}(-j),
\end{aligned}
$$

where $P_{B,-i, \mathfrak{Y}}^{\eta}(-j)=G r_{B, f, b, \mathfrak{Y}} \cap P_{B,-i}^{\eta}(-j)$. We apply Proposition 1.3.4 and obtain the splitting of mixed Hodge structures of the perverse filtration $\mathscr{P}_{B, f}$ :

$$
\phi_{\eta_{B}}: G r_{B, f} \stackrel{\cong}{\longrightarrow} I H_{B}(X)
$$

no matter which of the four decompositions (13) of $G r_{B, f}$ we plug in the l.h.s. 
1.4.2. The projectors associated with a projective map. We have the four direct sum decompositions stemming from (13) and (14):

$$
I H_{B}(X)=\bigoplus_{b} \phi_{\eta_{B}}\left(G r_{B, f, b}\right)=\bigoplus_{b, \mathfrak{Y}} \phi_{\eta_{B}}\left(G r_{B, f, b, \mathfrak{Y}}\right)=\cdots .
$$

Any direct summand of any direct sum decomposition above gives rise to the degree-preserving endomorphism of $I_{B}(X)$ obtained by projecting onto that summand. We thus obtain the Betti projectors of the decomposition theorem for $(f, \eta)$ :

$$
\begin{aligned}
& \pi_{B}(f, \eta, b), \pi_{B}(f, \eta, b, \mathfrak{Y}), \pi_{B}(f, \eta, i, j), \pi_{B}(f, \eta, i, j, \mathfrak{Y}) \\
\in & \left(\mathscr{W}_{0} \cap \mathscr{F}^{0}\right) \operatorname{End}^{o}\left(I_{B}(X)\right),
\end{aligned}
$$

where the inclusions in the appropriate steps of the filtrations $(\mathscr{W}, \mathscr{F})$ express that these projectors are maps of rational mixed Hodge structures.

\subsubsection{Decompositions for the composition of two proper maps.} We drop the Betti decoration. The purpose of this section is to clarify, via Lemma 1.4.1 below, the relation between the perverse filtrations arising from the maps $f, g, h$.

Let $Q$ be semisimple complex of geometric origin ([3]) on $W$. We have the direct sum decompositions $g_{*} Q \cong \bigoplus_{a}\left(\bigoplus_{\mathfrak{X}} Q_{g, a, \mathfrak{X}}\right)[-a]$ and, since $h_{*}=$ $f_{*} \circ g_{*}$ :

$$
h_{*} Q \cong \bigoplus_{c}\left(\bigoplus_{a+b=c} \bigoplus_{\mathfrak{X}, \mathfrak{Y}} Q_{g, a, \mathfrak{X} ; f, b, \mathfrak{Y}}\right)[-c]
$$

where it is understood that, for every fixed $(a, b)$, we take, for each support $\mathfrak{X} \in \mathcal{S}(g, a, Q)$, the supports $\mathfrak{Y} \in \mathcal{S}\left(f, b, Q_{g, a, \mathfrak{X}}\right)$.

We have the following perverse filtrations: $\mathscr{P}_{g}, \mathscr{P}_{h}$ on $H(W, Q)$ and $\mathscr{P}_{f}$ on $G r_{f, a}:=G r^{\mathscr{P}_{f}} H\left(X, Q_{f, a}\right)$. Clearly, $\mathscr{P}_{h}$ induces a filtration, still denoted by $\mathscr{P}_{h}$, on $G r_{f, a}$. Given $\mathfrak{X} \in \mathcal{S}(g, a, Q)$, consider the natural quotient map $q$ : $\mathscr{P}_{g, a} \rightarrow G r_{g, a}=\oplus_{\mathfrak{X}} G r_{g, a, \mathfrak{X}}$ and define $\mathscr{P}_{g, a, \mathfrak{X}}:=q^{-1} G r_{g, a, \mathfrak{X}}$. Clearly, $G r_{g, a, \mathfrak{X}}$ $=\mathscr{P}_{g, a, \mathfrak{X}} / \mathscr{P}_{g, a-1}$. Define $\mathscr{P}_{h, c, \mathfrak{Y}}$ and $\mathscr{P}_{f, b, \mathfrak{Y}}$ in a similar way.

The following lemma follows directly from the definitions and from (17). 
Lemma 1.4.1. We have the identity:

$$
\mathscr{P}_{f, b} G r_{g, a}=\mathscr{P}_{h, a+b} G r_{g, a}
$$

For every $\mathfrak{X} \in \mathcal{S}(g, a, Q)$ and $\mathfrak{Y} \in \mathcal{S}\left(f, b, Q_{g, a, \mathfrak{X}}\right)$, we have the identity:

$$
G r_{f, b, \mathfrak{Y}} G r_{g, a, \mathfrak{X}}=G r_{h, a+b, \mathfrak{Y}} G r_{g, a, \mathfrak{X}}=\frac{\mathscr{P}_{h, a+b, \mathfrak{Y}} \cap \mathscr{P}_{g, a, \mathfrak{X}}}{\mathscr{P}_{h, a+b-1} \cap \mathscr{P}_{g, a, \mathfrak{X}}}
$$

Remark 1.4.2. The relative hard Lefschetz theorem applied to $Q_{g, a} \mathscr{X}$ implies that the triple $\left(G r_{g, a, \mathfrak{X}}, \mathscr{P}_{f}, \eta\right)$ is an HL-triple (§1.3.4). We deduce the four decompositions of each $G r_{f} G r_{g, a, \mathfrak{X}}$ as in (13), as well as the splitting:

$$
\phi_{\eta}: G r_{f} G r_{g, a, \mathfrak{X}} \longrightarrow G r_{g, a, \mathfrak{X}}
$$

as in (14). When $Q=I C_{W}$, everything is compatible with the mixed Hodge structures in sight $([7])$. We are going to use this set-up in the special case when $g$ is a resolution of the singularities of $X$ integral, so that $G r_{g, 0, X}=$ $I H(X, \mathbb{Q})$.

\section{The projectors are absolute Hodge}

\subsection{The perverse filtration $\mathscr{P}_{f}$ is $K$-rational}

Proposition 2.1.1. Let $(f, \sigma)$ be as in 1). There is a unique filtration $\mathscr{P}_{f}$ of $H(X)$, which we name the perverse filtration associated with $f$, yielding compatibility diagrams $(*)$ for each subspace $\mathscr{P}_{f, b} H(X)$ and for each inclusion $\mathscr{P}_{f, b} H(X) \subseteq \mathscr{P}_{f, b^{\prime}} H(X)$ with $b \leq b^{\prime}$. At each Betti vertex, the filtration coincides with the Betti perverse filtration $\mathscr{P}_{B, f}$.

In particular, we can produce compatibility diagrams $(*)$ for $\mathscr{P}_{f}$ and for $G r_{f}:=G r^{\mathscr{P}_{f}}$ on $H(X)$ and on $H_{!}(X)$.

Proof. Given the nature of the arrows of diagram (3), unicity follows from the requirement that, at the Betti vertices, the filtration coincides with the Betti perverse filtrations.

As to the existence, we use the construction of $\S 1.3 .1$. Since the formation of the maps (7) is compatible with the arrows in (3), and strictly so for the filtrations $(\mathscr{W}, \mathscr{F})$, the filtrations $F^{\beta}$ correspond to each other via the arrows in (3). It follows that, for every $\beta \in \mathcal{B}(K)$, we have compatibility diagrams (*) for $H(X)$. Similarly for $F_{!}^{\beta}$ and $H_{!}(X)$. 
Recalling that given a $K$-scheme $Z$, the set $Z(K)$ of its $K$-rational points is dense in every $\sigma Z(\mathbb{C})$, Fact 1.3.1 implies immediately that, for $\beta \in \mathcal{B}(K)$ generic, the filtrations of type $F_{B}^{\beta}$ and $F_{!, B}^{\beta}$ appearing in every Betti vertex of $(3)$ for $H(X)$ and $H_{!}(X)$ coincide with the corresponding perverse filtrations $\mathscr{P}_{B, f}$.

\section{2. $X$ nonsingular: the supports and the maps (13), (14) are $\boldsymbol{K}$-rational}

The goal of this section is to prove Proposition 2.2.1, stating that, when $X$ is nonsingular, the support and primitive decompositions (13), (14) fit in a diagram $(*)$.

Proposition 2.2.1. Let $(f, \eta, \sigma)$ be as in 1$)$. Assume that $X$ is nonsingular. There are decompositions 13) and splittings 14) giving rise to corresponding compatibility diagrams $(*)$ in cohomology and in cohomology with compact supports.

Proof. The proof proceeds by induction on $m:=\operatorname{dim} X$. We assume that the proposition holds for every proper map $g: Z \rightarrow Z^{\prime}$ of quasi-projective varieties with $Z$ nonsingular and $\operatorname{dim} Z<\operatorname{dim} X$. Note that if $\operatorname{dim} Z=0$, then the inductive hypothesis is trivial and the conclusion of the proposition is trivially true.

Let $\mathscr{T}_{Y}$ be a stratification of $Y$ as in Proposition 1.3.2, so that, for every $\sigma$, the supports of the $\mathbb{C}$-map $\sigma f$ are among the closures of the strata of $\sigma \mathscr{T}_{Y}$.

For every non-dense $T \in \mathscr{T}_{Y}$, form the $K$-diagram (8) for the map $f$. If necessary, we refine the stratification so that it satisfies the conclusion of Proposition 1.3.2 for $g$ as well.

Since $\operatorname{dim} \mathcal{T}<\operatorname{dim} X$, we can apply the inductive hypothesis to $g$ : we have decompositions and splittings (13) and (14) for $G r_{g, b}$ and $G r_{!, g, b}$, giving rise to compatibility diagrams $(*)$. The fact that $\mathcal{T}$ may fail to be integral does not effect the arguments in a substantial way.

Since $r$ is a $K$-map, we have compatibility diagram $(*)$ for the maps $r^{*}$ and $r_{!}^{*}$ which, up to the shift of filtration discussed in $\S 1.3 .3$, are compatible with the respective perverse filtrations: this is the case at the Betti vertices, and is thus automatic at all the other vertices.

By denoting, as in Section 1.3.3, by $r_{b, \bar{T}}^{*}: G r_{f, b} H(X) \rightarrow G r_{g, b^{\prime}, \bar{T}} H(\mathcal{T})$ the map of graded pieces induced by $r^{*}$, followed by the projection onto the 
$\bar{T}$-th direct summand of $G r_{g, b^{\prime}}$, we have compatibility diagram (*) for the maps $r_{b, \bar{T}}^{*}$. Similarly, for $r_{!, b, \bar{T}}^{*}$.

Since we have the compatibility diagram $(*)$ for the Poincaré pairing on $X$, the usual argument -validity of a given assertion at the Betti vertex - coupled with Proposition 2.1.1, ensures that we have the compatibility diagram $(*)$ for the graded version $(10)$ of the pairing.

We can therefore define the summands of the decomposition by supports of $H(X)$ at every vertex of the compatibility diagram (*) by using the equalities (12) and (11) in the three cohomology theories. The resulting decompositions by supports give rise to the compatibility diagrams (*) automatically.

Since the primitive decompositions are defined via the linear algebra properties of cupping with $\eta$, the same is true for all four decompositions in (13).

Finally, the compatibility diagram $(*)$ for the splitting (14) is obtained formally by applying Proposition 1.3.4 to each vertex of the diagram.

\subsection{K-rational intersection de Rham cohomology}

Proposition 2.3.1. Let $X$ be a quasi-projective $K$-scheme. We have a natural compatibility diagram $(*)$ for $I H(X)$ and for $I_{!}(X)$.

Proof. We may assume that $X$ is reduced. Let $g: W \rightarrow X$ be a "resolution" of the singularities of $X$, i.e. $W$ is nonsingular and each irreducible component of $W$ is a resolution of the singularities of an irreducible component of $X$. We use the notation of $\S 1.4 .3$. Note that $I C_{X}$ is the direct summand $Q_{g, 0, X}$ of $g_{*} I C_{W}$ in perversity 0 .

Let $\mathscr{P}_{g}$ be the perverse filtration for $g$ on $H(W)$. We apply Proposition 2.2.1 to $g$ and obtain the compatibility diagram (*) for $G r_{g, 0, X}$. It only remains to define the intersection de Rham cohomology groups of $X$ as the $K$-vector spaces:

$$
I H_{d R}(X / K):=G r_{g, 0, X} H_{d R}(W / K),
$$

and similarly for $I H_{!, d R}(X / K)$.

It is a routine matter to verify that these bifiltered groups (they are endowed with the weight and the Hodge filtrations) are independent of the resolution chosen to define them; see [10], Theorem 2.2.3.a. Here is a partial list of useful properties enjoyed by these groups (verifications left to 
the reader; $X$ integral for simplicity): non-degenerate intersection pairing in intersection cohomology; map from cohomology to intersection cohomology; map $I H_{!, d R}(X / K) \rightarrow I H_{d R}(X / K)$; cup product with de Rham cohomology $H_{d R}^{i}(X / K) \otimes I H_{d R}^{j}(X / K) \rightarrow I H_{d R}^{i+j}(X / K)$; cycle classes; restrictions and Gysin maps for normal nonsingular inclusions ([10], p. 714]); restrictions to Zariski open subvarieties.

\section{4. $X$ possibly singular: the maps (13) and (14) are $K$-rational}

This section can be skipped if in Theorem 2.5.1 we assume that $X$ is nonsingular.

Let things be as in (1). In the Betti context, if we set $Q:=I C_{W}$, then we have to the decompositions of $G r_{f} G r_{g, a, \mathfrak{X}} H(W)$ and the splitting (20) of Remark 1.4.2.

Proposition 2.4.1. Let things be as in 1) with $W$ nonsingular. We have compatibility diagrams (*) for the decompositions and splittings of Remark 1.4.2

Proof. Since $W$ is nonsingular, Propositions 2.1.1 and 2.2.1 allow us to form the compatibility diagrams $(*)$ for the r.h.s. of the two equalities (18) and (19) of Lemma 1.4.1. The conclusion follows.

\subsection{The projectors of the decomposition theorem are absolute Hodge}

Let things be as (1), with $g$ a resolution of the singularities of $W$. We apply Proposition 2.4.1 in the case of $G r_{g, 0, X}$, which gives us the compatibility diagram $(*)$ for $I H(X)$ (Proposition 2.3.1). Proposition 2.4.1 applies and we obtain compatibility diagrams $(*)$ for the decompositions of type (13) of $G r_{f} G r_{g, 0, X}$ given in Remark 1.4.2, and for the splitting $\phi_{\eta}(20)$ in the same remark. From now on, we write $I H(X)$ and $G r_{g, 0, X}$ interchangeably.

We define projectors $\pi(f, \eta, b)$ at each vertex of the compatibility diagram $(*)$ for $\operatorname{End}^{o}(I H(X))$ by taking the projection onto the summand $\phi_{\eta}\left(G r_{f, b} G r_{g, 0, X}\right)$ of $I H(X)=G r_{g, 0, X}$ associated with $\phi_{\eta}$. Form the compatibility diagram $(*)$ associated with $\operatorname{End}^{o}(I H(X))$ and form the endomorphisms:

$$
\underline{\pi}(f, \eta, b) \in \underline{\operatorname{End}}^{o}(I H(X)) .
$$


The collection $\underline{\pi}(f, \eta, b), b \in \mathbb{Z}$ is a complete system of orthogonal projectors:

$$
\underline{\pi}(f, \eta, b) \circ \underline{\pi}\left(f, \eta, b^{\prime}\right)=\delta_{b b^{\prime}} \underline{\pi}(f, \eta, b), \quad \sum_{b} \underline{\pi}(f, \eta, b)=\underline{\operatorname{Id}}_{I H(X)} .
$$

We can do the same for the remaining decompositions in (13) and obtain projectors $\underline{\pi}(f, \eta, i, j), \underline{\pi}(f, \eta, b, \mathfrak{Y})$ and $\underline{\pi}(f, \eta, i, j, \mathfrak{Y})$. These projectors are related in the following way: they refine each other in the same way the decompositions (13) refine each other.

We have the analogue $\underline{\pi}$ ! for compact supports.

Theorem 2.5.1. Let $(f, \eta, K)$ be as in (1). The projectors (22) and their compact supports analogue are absolute Hodge.

Proof. We have the compatibility diagram (*) for $\operatorname{End}^{o}(I H(X))$. Fix one of the four kind of projectors in $\operatorname{End}^{\circ}(I H(X))$ and call it $\pi$. By the very definition of $\pi$ and by Proposition 2.4.1, we have that the $\pi$ 's at each vertex correspond to each other via the arrows of the diagram. Since the projector is defined in $\mathbb{Q}$-Betti cohomology, we have that $\sigma^{*} \underline{\pi}$ is rational. Since the projectors are (strictly) compatible with $(\mathscr{F}, \mathscr{W})$, we have that $\pi_{d R} \in\left(\mathscr{F}^{0} \cap\right.$ $\left.\mathscr{W}_{0}\right) \operatorname{End}^{o}(I H(X))$. The compact supports analogue is proved in the same way.

Remark 2.5.2. Let $X_{1} \stackrel{f_{1}}{\longrightarrow} X_{2} \stackrel{f_{2}}{\longrightarrow} \cdots \stackrel{f_{n-1}}{\longrightarrow} X_{n} \stackrel{f_{n}}{\longrightarrow}$ be proper maps of quasiprojective $K$-schemes. Set $g_{i}:=f_{i} \circ \cdots \circ f_{1}$. We leave to the reader the task to formulate and prove by induction on $n$ that there is a natural $n$-tupleversion of the first two decompositions (13), involving multi-supports of the multi-graded pieces $G r_{b_{n}}^{g_{n}} \cdots G r_{b_{1}}^{g_{1}} I H\left(X_{1}\right)$; see $\S 1.4 .3$ for a layout of the case $n=2$. There is the further variant, where at each stage one takes the primitive decompositions associated with choices of $f_{i}$-ample line bundles. The reader can formulate and prove the $n$-tuple variant of Theorem 2.5.1 for this situation.

Remark 2.5.3. The paper [8] constructs five distinct distinguished isomorphisms of type (14), each yielding a collections of projectors as above. Theorem 2.5.1 remains valid in each of these variants and the proofs are similar. While the five collections of projectors are distinct, the resulting five collections of absolute Hodge motives are mutually isomorphic. 


\subsection{The projectors are André motivated for $X$ projective nonsingular}

The content of this section is the result of discussions with F. Charles. We place ourselves in the context of $\S 1.2 .1: \mathcal{M}_{K}$ is the category of André motives over the algebraically closed field of characteristic zero $K$. We aim at proving Theorem 2.6.2. We need a preliminary lemma.

Lemma 2.6.1. Let $r: X^{\prime} \longrightarrow X$ be a map of nonsingular $K$-varieties with $X$ projective. There is a natural subobject $k_{r} \subseteq h(X)$ in $\mathcal{M}_{K}$ whose de Rham realization is $\operatorname{Ker} r^{*} \subseteq H_{d R}(X / K)$.

Proof. The methods of [14] endow de Rham (co)homology with the weight and Hodge filtrations, and pull-backs and Gysin maps are filtered strict for both. This can be seen by descending the situation from $K$ to a suitable subfield embeddable into $\mathbb{C}$, by base changing to $\mathbb{C}$, and then by using the bifiltered strict comparison isomorphisms with the Betti theory, which enjoys the desired properties. We are thus free to use the usual properties of weights as in [14].

Choose a commutative diagram of quasi-projective $K$-schemes:

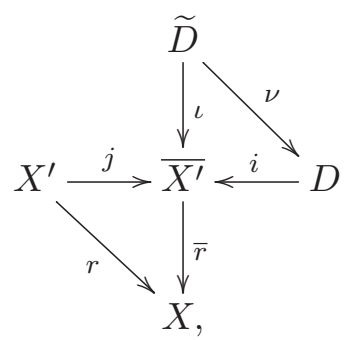

where $j$ is a smooth projective compactification with simple normal crossing divisor $D ; \nu$ is a resolution of the singularities of $D$ (e.g. the normalization); the map $r$ extends to a map $\bar{r}$ (compactify, resolve, put in normal crossing).

By using the de Rham analogue of Lefschetz duality, we get, for every $k \geq 0$, the following commutative diagram (some decorations omitted; $d^{\prime}:=$ $\operatorname{dim} X^{\prime}$ ), where the horizontal line is exact (long exact sequence of relative cohomology); the arrows are filtered strict for the Hodge and weight filtrations.

Clearly, $\operatorname{Ker} r^{*}=\bar{r}^{*-1}\left(\operatorname{Ker} j^{*}\right)=\bar{r}^{*-1}\left(\operatorname{Im} i_{*}\right)$. 


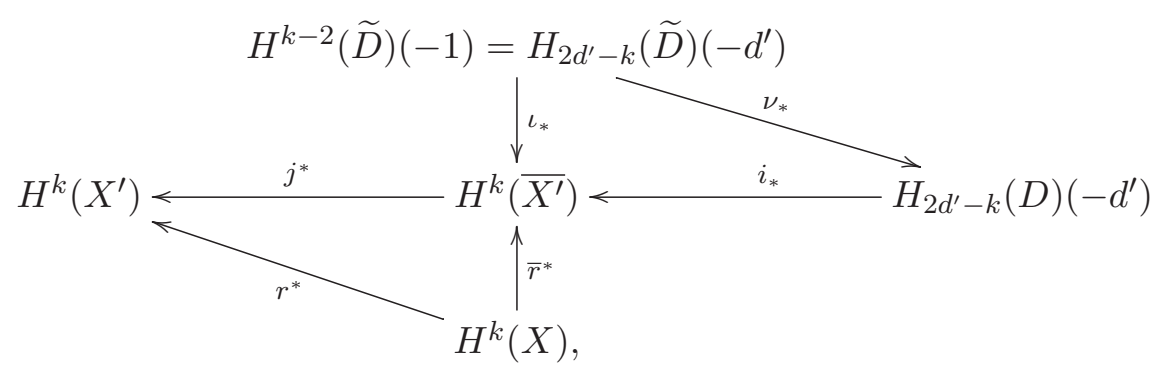

CLAIM. We have $\operatorname{Im} i_{*}=\operatorname{Im} \iota_{*}$, so that, obviously, $\operatorname{Ker} r^{*}=\bar{r}^{*-1}\left(\operatorname{Im} \iota_{*}\right)$.

Proof of the CLAIM. By mixed Hodge theory, the map $\nu_{*}$ is a surjection onto the lowest-weight part $\mathscr{W}_{k} H_{2} \operatorname{dim} \mathcal{X}-k D\left(-\operatorname{dim} X^{\prime}\right)$. The image of $i_{*}$ is of pure weight $k$ so that, by strictness, it comes from $\mathscr{W}_{k} H_{2} \operatorname{dim} \mathcal{X}-k D\left(-\operatorname{dim} X^{\prime}\right)$ and the CLAIM follows.

The category $\mathcal{M}$ is abelian, so that we can form the compositum:

$$
v: h(X) \stackrel{h(\bar{r})}{\longrightarrow} h\left(\overline{X^{\prime}}\right) \longrightarrow h\left(\overline{X^{\prime}}\right) / \operatorname{Im} h(\iota) .
$$

In view of the CLAIM, the kernel Ker $v$ in $\mathcal{M}$ has de Rham realization Ker $r^{*}$.

Using standard techniques (take a common resolution of two choices), one shows easily that $\operatorname{Ker} v$ is well-defined up to canonical isomorphism independently of the choices made in the initial commutative diagram.

Theorem 2.6.2. Let $(f, \eta, K)$ be as in 1$)$ with $K$ algebraically closed of characteristic zero and with $X$ nonsingular and projective. The projectors of the decomposition theorem (§2.5) are motivated cycles in $A_{\mathrm{mot}}^{\operatorname{dim} X}(X \times X)$.

Proof. With Lemma 2.6.1 in hand, the proof of Theorem 2.6.2 is virtually identical to the one of Theorem 2.5.1, which we now review briefly in the context of $\mathcal{M}_{K}$.

Lemma 2.6.1 and Proposition 2.1.1 give us motives whose de Rham realization is the perverse filtration $\mathscr{P}_{f} H_{d R}(X)$. We denote these motives by $h\left(\mathscr{P}_{f}\right)$. Note the misleading potential of the notation: we are not applying $h$ to form this motive, but, rather, we are using Lemma 2.6.1 to define it. The role of the lemma is precisely to circumvent the fact that in the geometric description of the perverse filtration recalled in Proposition 2.1.1, one does not immediately "promote" diagram (6) to the category $\mathcal{M}_{K}$ because we 
are not aware of a theory of André motives for nonsingular open varieties, here the elements of the flag $X_{\bullet}^{\prime}$ in said diagram.

We now produce motives $h\left(G r_{f}\right)$, decompositions of $h\left(G r_{f}\right)$ as in (13), the splitting (14) and, finally, motivated projectors $\pi$ in $\operatorname{End}(h(X))=$ $A_{\text {mot }}^{\operatorname{dim} X}(X \times X)$ which map, via $c l_{d R}$, to our de Rham projectors of the decomposition theorem in $H_{d R}^{2 \operatorname{dim}} X(X \times X)(\operatorname{dim} X)$.

Remark 2.6.3. Given Theorem 2.6.2, one can define a canonical André motive attached to the intersection cohomology groups of any projective $K$-scheme $X$ by choosing a resolution $g: W \rightarrow X$, and defining it as in Equation (21), which makes sense in the category $\mathcal{M}_{K}$. The verification that this notion is independent of the resolution is standard, and so is the verification of the formal properties stated in $\S 2.3$. The reader should have no problems in applying the methods of this paper to prove that Theorem 2.5.1, as well as its variants outlined in Remarks 2.5.2, 2.5.3, have an intersection cohomology counterpart in $\mathcal{M}_{K}$.

Remark 2.6.4. As the referee has kindly pointed out, M. Saito's theory of mixed Hodge modules in an arithmetic context [23], allows to construct absolute Hodge motives whose Betti realizations are the intersection cohomology groups.

Remark 2.6.5. In the paper [5], A. Corti and M. Hanamura show that, if the standard conjectures hold, then it is possible to upgrade the decomposition theorem to the level of motives and, in particular, to define Chow motives whose Betti realizations are the intersection cohomology groups. In [9] it is shown that the projectors on the summands corresponding to the different supports of a semismall map, but not the projectors onto a given cohomological degree, are given by algebraic cycles. The result is generalized to generically finite maps of threefolds in [11]. Some new special cases could arise from recent progress on the standard conjectures (e.g. [4]).

\section{Variants of Theorem 2.5.1}

\subsection{The projectors are absolute Hodge over an algebraically closed field of characteristic zero}

Theorem 2.5.1 is stated for algebraically closed fields with finite transcendence degree over the rationals. In [15] the notion of Absolute Hodge class, or map, is first introduced for algebraically closed fields of characteristic zero 
with finite transcendence degree over the rationals; it is then extended to arbitrary algebraically closed fields of characteristic zero by descending to algebraically closed subfields of finite transcendence degree over the rationals; finally, it is extended to arbitrary fields of characteristic zero by passing to an algebraic closure and by considering Galois invariants. It is thus clear that a class, or map, defined over any algebraically closed field of characteristic zero, is absolute Hodge iff it can be descended to an absolute Hodge class, or map, over an algebraically closed subfield of finite transcendence degree over the rationals. It is also clear that the constructions in $\S 1.3$ are of this nature.

It follows that Theorem 2.5.1 holds over any algebraically closed field of characteristic zero and that given a field of characteristic zero, it holds over a suitable finite extension.

We have defined the groups $I H_{d R}(X / K)$ (21) assuming $K$ algebraically closed of finite type over $\mathbb{Q}$. What above shows that one can do so over any field of characteristic zero.

\subsection{The projectors are Tate classes}

Let $K_{0}$ be a field of arbitrary characteristic, with a fixed algebraic closure $K_{0} \subseteq K$. Let $X_{0}$ be a $K_{0}$-scheme and let $X$ be the resulting $K$-scheme. For every prime number $\ell \neq$ char $K$, the $\mathbb{Q}_{\ell}$-adic cohomology (with and without compact supports) of $X$ carries a continuous action of the profinite group $\operatorname{Gal}\left(K / K_{0}\right)$.

We say that an element in some $H_{e t}\left(X, \mathbb{Q}_{\ell}\right)(m)$, or in $\operatorname{End}^{o}\left(H_{e t}\left(X, \mathbb{Q}_{\ell}\right)\right.$, is of Tate type if it is $\operatorname{Gal}\left(K / K_{0}\right)$-invariant. Similarly, for compact supports.

Let $(f, \eta)$ be as in $(1)$, except that we work over an arbitrary, i.e. not necessarily algebraically closed field $K$. The methods of this paper show that the projectors of the decomposition theorem, defined in $\mathscr{W}_{0} \operatorname{End}^{o}\left(I_{\text {et }}\left(X \otimes_{K}\right.\right.$ $\left.\bar{K}, \mathbb{Q}_{\ell}\right)$ ) as in (16), after passing to an algebraic closure $\bar{K}$ of $K$, are invariant under the action of the group $\operatorname{Gal}\left(\bar{K} / K_{1}\right)$, where $K_{1}$ is a suitable finite extension of $K$. In particular, said projectors are Tate for the situation over $K_{1}$.

According to the Tate conjecture, if $K$ is finitely generated over it prime field, and $X$ is geometrically irreducible smooth and projective, then these projectors are expected to be algebraic.

\subsection{The projectors are absolutely Hodge and Tate in the sense of Ogus}

In this section, we adapt A. Ogus' definitions [22] of absolutely Hodge and absolutely Tate de Rham classes to the case of maps, and prove that the 
projectors in the decomposition theorem have this property. Since we work with crystalline cohomology for varieties over a perfect field of positive characteristic (see the survey [21]) and this theory is not well-behaved for either singular, or non proper varieties, we place ourselves in the safer smooth and proper niche. Alternatively, one may work with rigid cohomology.

Let $K$ be a field of characteristic zero, let $R \subseteq K$ be a smooth $\mathbb{Z}$-algebra, set $\mathcal{R}:=\operatorname{Spec} R$ and let $\mathcal{X} \rightarrow \mathcal{R}$ be a proper and smooth map. It is standard that a proper smooth $X / K$-scheme can be descended to such an $\mathcal{X} / R$ and that the closed points of $\mathcal{R}$ have finite, hence perfect, residue fields. Let $c \in \mathcal{R}(\mathbb{C})$ be a $\mathbb{C}$-point of $\mathcal{R}$. Denote by $\mathcal{X}_{c}$ the $\mathbb{C}$-variety obtained by base change. We have the natural maps:

$$
c^{*}: H_{d R}(\mathcal{X} / R) \longrightarrow H_{d R}\left(\mathcal{X}_{c} / \mathbb{C}\right) \stackrel{\cong}{\longrightarrow} H_{B}\left(\mathcal{X}_{c}, \mathbb{C}\right) .
$$

Let $k$ be a perfect field of char $\mathrm{k}=\mathrm{p}>0$. Let $W=W(k)$ be the ring of Witt vectors of $k$. Let $\sigma \in \mathcal{R}(W)$ be a $W$-point of $\mathcal{R}$ and let $\bar{\sigma} \in \mathcal{R}(k)$ be the resulting $k$-point, i.e. the compositum: $R \rightarrow W \rightarrow k$. We get the Cartesian diagram:

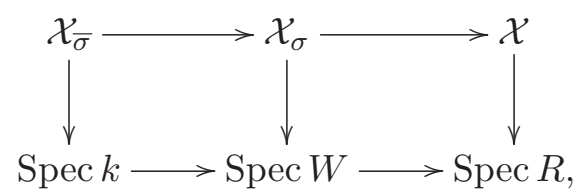

with resulting maps (de Rham/crystalline comparison: $\mathbb{K}:=$ the quotient field of $W)$ :

$$
\bar{\sigma}^{*}: H_{d R}(\mathcal{X} / R) \longrightarrow H_{d R}\left(\mathcal{X}_{\sigma} / W\right) \otimes_{W} \mathbb{K} \stackrel{\cong_{\mathbb{K}}}{\longrightarrow} H_{\text {cris }}\left(\mathcal{X}_{\bar{\sigma}} / W\right) \otimes_{W} \mathbb{K}
$$

The crystalline Frobenius is the automorphism of the rhs of (26) induced by the action of absolute Frobenius on $\mathcal{X}_{\bar{\sigma}}$.

Definition 3.3.1. Let $X$ be smooth and proper over a field $K$ of characteristic zero. Let $\xi \in \operatorname{End}^{o}\left(H_{d R}(X / K)\right)$. We say that $\xi$ is absolutely Hodge, if there are $\mathcal{X} / R$ as above inducing $X / K$, and $\xi_{R} \in \operatorname{End}^{o}\left(H_{d R}(\mathcal{X} / R)\right)$ inducing $\xi$, such that, for every $c \in \mathcal{R}(\mathbb{C}), c^{*} \xi_{R}$ is a Hodge class. We say that $\xi$ is absolutely Tate, if there are $\mathcal{X} / R$ and $\xi_{R}$ as above, such that, for every $W$ and every $\sigma \in \mathcal{R}(W), \bar{\sigma}^{*} \xi_{R}$ is invariant under crystalline Frobenius.

Theorem 3.3.2. Let $(f, \eta)$ be as in (1), except that we assume that $X, Y$ are projective, $X$ is smooth and $K$ is a field of characteristic zero. After 
passing to a suitable finite extension of $K$, the projectors of the decomposition theorem are defined and absolutely Hodge and Tate in the sense of Ogus.

Sketch of proof. The discussion in $§ 3.1$ implies that the projectors are defined after passing to a suitable finite extension of $K$. We replace $K$ with such an extension. The same discussion in $\S 3.1$ shows that the projectors are in fact defined over a suitable smooth $\mathbb{Z}$-algebra $R \subseteq K$ over which the whole situation descends, with $\mathcal{X} / R$ smooth and projective.

Ogus' density argument [22], Remark 4.5 ensures that in order to prove the "absolutely Hodge" part of the statement it is enough to verify it at a $\mathbb{C}$ point supported at the generic point of $\mathcal{R}$. This, in turn, follows immediately from our Theorem 2.5.1.

We turn to the "absolutely Tate" part of the statement.

As it is recalled in $\S 3.1$, the projectors in de Rham cohomology are constructed via diagrams (6), (8) and the splitting $\phi_{\eta}(20)$. In view of the functoriality properties of crystalline cohomology in the context of smooth and proper varieties, the use of the second and third ingredient can be carried out in crystalline cohomology, compatibly with the de Rham/crystalline comparison isomorphism (26). On the other hand, the first one involves nonproper varieties and is thus problematic on the crystalline side. This issue is easily by-passed via the CLAIM in the proof of Lemma 2.6.1: one enlarges $R \subseteq K$ by adding finitely many elements so that diagram (23) descends to it. If necessary, one inverts an element to ensure smoothness of $\mathcal{X} / R$. We are now free to form the filtration in $H_{\text {cris }}\left(X_{\bar{\sigma}} / W\right)$ via the use of diagram (6) base-changed to the perfect field $k$, compatibly with the comparison isomorphism (26). It follows that the formation of every object leading to the definition of the projectors in crystalline cohomology is now crystalline Frobenius-invariant.

\section{Acknowledgements}

We thank F. Charles and to B. Bhatt for useful conversations. We thank the anonymous referee for the thoughtful suggestions. Mark Andrea de Cataldo partially supported by N.S.A. grant H982300910068 and N.S.F. grants DMS 0907826 and 1301761. Luca Migliorini partially supported by PRIN project 2012 "Spazi di moduli e teoria di Lie". 


\section{References}

[1] Y. André, Pour une théorie inconditionelle des motifs. Publ. I.H.E.S., 83 (1996), 5-49.

[2] D. Arapura, An abelian category of motivic sheaves. Advances in Mathematics, 233(1), 135-195.

[3] A. A. Beilinson, J. N. Bernstein and P. Deligne, Faisceaux pervers. Astérisque, 100, Soc. Math. Fr., Paris, 1982.

[4] F. Charles and E. Markman, The Standard Conjectures for holomorphic symplectic varieties deformation equivalent to Hilbert schemes of K3 surfaces. Comp. Math., 149 (2013), no. 3, 481-494.

[5] A. Corti and M. Hanamura, Motivic decomposition and intersection Chow groups. I. Duke Math. J., 103 (2000), no. 3, 459-522.

[6] M. A. de Cataldo, The standard filtration on cohomology with compact supports with an appendix on the base change map and the Lefschetz hyperplane theorem. Interactions of classical and numerical algebraic geometry, 199-220, Contemp. Math., 496, Amer. Math. Soc., Providence, RI, 2009.

[7] M. A. de Cataldo, The perverse filtration and the Lefschetz Hyperplane Theorem, II. J. Algebraic Geom., 21 (2012), no. 2, 305-345.

[8] M. A. de Cataldo, Hodge-theoretic splitting mechanisms for projective maps. With an appendix containing a letter from P. Deligne. J. Singul., 7 (2013), 134-156.

[9] M. A. de Cataldo and L. Migliorini, The Chow motive of semismall resolutions. Math. Res. Lett., 11 (2004), 151-170.

[10] M. A. de Cataldo and L. Migliorini, The Hodge Theory of Algebraic maps. Ann. Scient. Éc. Norm. Sup., $4^{e}$ série, t., 38 (2005), 693-750.

[11] M. A. de Cataldo and L. Migliorini, Intersection forms, algebraic maps and motivic decomposition for resolution of threefolds. In: Algebraic Cycles and Motives, Vol. 1, London Math. Soc. Lecture Note Series, 343, 102-137, Cambridge University Press, Cambridge, UK, 2007.

[12] M. A. de Cataldo and L. Migliorini, The decomposition theorem, perverse sheaves and the topology of algebraic maps. Bulletin of the Amer. Math. Soc., 46 (2009), 535-633. 
[13] M. A. de Cataldo and L. Migliorini, The perverse filtration and the Lefschetz hyperplane Theorem. Annals of Math., 171 (2010), no. 3, 20892113.

[14] P. Deligne, Théorie de Hodge, III. Publ. Math. IHES, 44 (1974), 5-78.

[15] P. Deligne, Hodge cycles on Abelian varieties. In: Hodge cycles, Motives and Shimura varieties, Lect. Notes in Math., 900, Springer Verlag, 1982. (We actually use the updated version: http://www.jmilne.org/math/ Documents/Deligne82.pdf)

[16] P. Deligne, Décompositions dans la catégorie Dérivée. Motives (Seattle, WA, 1991), 115-128, Proc. Sympos. Pure Math., 55, Part 1, Amer. Math. Soc., Providence, RI, 1994.

[17] P. Deligne and J. S. Milne, Tannakian Categories. In: Hodge cycles, Motives and Shimura varieties, Lect. Notes in Math., 900, Springer Verlag, 1982.

[18] M. Goresky and R. MacPherson, Stratified Morse Theory. Ergebnisse der Mathematik, und ihrer Grenzgebiete 3. Folge. Band 2, SpringerVerlag, Berlin-Heidelberg, 1988.

[19] A. Grothendieck, On the de Rham cohomology of algebraic varieties. Publ. Math. IHES, 29 (1966), 351-359.

[20] R. Hartshorne, On the de Rham cohomology of algebraic varieties. Publ. Math. IHES, 45 (1975), 5-99.

[21] L. Illusie, Report on crystalline cohomology. In: Algebraic GeometryArcata 1974, Proc. Symp. in Pure Mathamatics, Volume XXIX, 459478, A.M.S. Providence RI 1975.

[22] A. Ogus, Hodge cycles and crystalline cohomology. In: Hodge cycles, Motives and Shimura varieties, Lect. Notes in Math. 900, 357-414, Springer Verlag, 1982.

[23] M. Saito, Arithmetic mixed sheaves. Invent. Math., 144 (2001), no. 3, $533-569$.

[24] B. Teissier, Variétés polaires. II. Multiplicités polaires, sections planes, et conditions de Whitney. Algebraic geometry (La Rabida, 1981), 314491, Lecture Notes in Math., 961, Springer Berlin, 1982.

[25] J. L. Verdier, Stratifications de Whitney et Théorèmes de Bertini-Sard. Invent. Math., 36 (1976), 295-312. 
Department of Mathematics, Stony Brook University STONY BRoOK, 11794-3651, NY, USA

E-mail address: mark.decataldo@stonybrook.edu

Dipartimento di Matematica, Università di Bologna

Piazza Porta S. Donato, 5, 40126, Bologna, Italy

E-mail address: luca.migliorini@unibo.it

Received August 21, 2014 